02,13

\title{
Зависимость критической температуры цилиндрических сверхпроводников от граничных условий
}

\author{
(C) А.Н. Лыков \\ Физический институт им. П.Н. Лебедева РАН, \\ Москва, Россия \\ E-mail: lykovan@lebedev.ru \\ Поступила в Редакцию 14 мая 2020 г. \\ В окончательной редакции 14 мая 2020 г. \\ Принята к публикации 15 мая 2020 г.
}

\begin{abstract}
Представлены результаты исследования свойств длинных цилиндрических сверхпроводников диаметром порядка длины когерентности $\xi$, проведенные в рамках теории Гинзбурга-Ландау (ГЛ). При решении уравнения ГЛ использовались граничные условия общего вида на параметр порядка. Использование таких граничных условий позволяет учесть влияние границы цилиндра на его сверхпроводящие свойства. Такой учет важен для цилиндров малого диаметра, свойства которых ощутимо зависят от свойств их границ.
\end{abstract}

Ключевые слова: сверхпроводящий цилиндр, критическая температура, граничные условия, теория Гинзбурга-Ландау.

DOI: 10.21883/FTT.2020.10.49901.108

\section{1. Введение}

Макроскопическая теория ГЛ [1] имеет важное значение для изучения свойств сверхпроводящих структур различной геометрии [2,3]. При этом развитие компьютерной техники дает возможность находить численными методами точные решения системы уравнений ГЛ для ряда сверхпроводящих систем [4-12], что позволяет лучше понять процессы, происходящие в реальных структурах из сверхпроводящих материалов. Естественно, при решении уравнений ГЛ, которые являются дифференциальными уравнениями второго порядка, важное значение имеют граничные условия. Особенно это важно для объектов малых размеров, поскольку их свойства во многом зависят от свойств их границ. Первоначально влияние граничных условий изучалось на свойства тонких сверхпроводящих пластин. При этом решались одномерные уравнения ГЛ в декартовых координатах, для которых граничное условие общего вида на параметр порядка $(\Psi)$ было получено в $[13,14]$, и оно имеет вид

$$
\begin{aligned}
& \partial \Psi / \partial x=1 /\left.\Lambda \Psi\right|_{x=-d / 2}, \\
& \partial \Psi / \partial x=-1 /\left.\Lambda \Psi\right|_{x=d / 2},
\end{aligned}
$$

где $d-$ толщина пленки, $\Lambda$ - феноменологический коэффициент размерности длины, иногда называемый длиной экстраполяции (коэффициент $\Lambda$ определяется свойствами материала, с которым граничит сверхпроводник). В случае обычных низкотемпературных сверхпроводников для их границы с диэлектриком обычно полагается, что $1 / \Lambda=0$, то есть $\Lambda \rightarrow \infty$. В работах $[14,15]$ было показано, что в отсутствие магнитного поля и тока условие (1) с конечной длиной экстраполяции приводит к уменьшению критической температуры тонких сверхпроводящих пленок. Толщина пленок $\left(d=d_{c}(T)\right)$, при которой исчезает сверхпроводимость, определяется следующим условием:

$$
d_{c}(T)=2 \xi(T) \arctan \frac{\xi(T)}{\Lambda},
$$

где $\xi(T)$ - зависящая от температуры длина когерентности в теории ГЛ. Очевидно, что выражение (2) определяет в неявном виде зависимость $T_{c}(d)$, которое, как правило, используется в аналитических и численных расчетах.

Было бы интересно узнать, как влияет матрица из несверхпроводящего материала на сверхпроводящие свойства сверхпроводника цилиндрической формы с малым диаметром. Данная задача имеет важное значение поскольку промышленные сверхпроводящие кабели с рекордными критическими токами имеют такую же структуру: сверхпроводниковых нитей малого диаметра в матрице из нормального металла [16].

\section{2. Формулировка задачи}

В работе [6] изучались свойства цилиндра в параллельном его оси магнитном поле на основе численного решения одномерных уравнений ГЛ в цилиндрических координатах с обычными граничными условиями, когда в соотношении (1) $1 / \Lambda=0$. В данной работе решалось уравнение ГЛ для параметра порядка также в цилиндрических координатах, но с новыми граничными условиями для случая длинного сверхпроводящего цилиндра радиусом $R$. В отсутствие магнитного поля и для однородного сверхпроводника цилиндрической формы получается одномерное уравнение для параметра порядка, в котором присутствует только зависимость 
параметра порядка от радиальной координаты $(r)$. В приведенных единицах уравнение имеет следующий вид:

$$
\frac{\partial^{2} \psi}{\partial \rho^{2}}+\frac{\partial \psi}{\rho \partial \rho}+\psi-\psi^{3}=0
$$

где $\rho=r / \xi, \psi=\Psi / \Psi_{0}, \Psi_{0}$ - параметр порядка в глубине сверхпроводника при нулевом внешнем магнитном поле.

Для учета влияния границы сверхпроводника, например, в случае сверхпроводникового цилиндра в матрице из нормального металла, граничное условие принимает вид [12]:

$$
\frac{\partial \psi}{\xi \partial \rho}=-\left.\frac{\psi}{\Lambda}\right|_{\rho=R / \xi}
$$

Отметим, что при $\Lambda=\infty$ реализуется обычно используемое граничное условие в теории ГЛ.

Длина когерентности $\xi$ зависит от температуры, поэтому приведенное выражение является неявной функцией температуры и формально справедливо при любой температуре $T$. Однако сами уравнения ГЛ применимы лишь в пределе $T \rightarrow T_{\mathrm{cm}}\left(T_{\mathrm{cm}}-\right.$ критическая температура массивного сверхпроводника, из которого изготовлен цилиндр). Остановимся более подробно на области применимости теории ГЛ. Формально такое условие формулируется в виде $T_{\mathrm{cm}}-T \ll T_{\mathrm{cm}}$. При этом существуют примеры когда формулы и зависимости, полученные в этом предельном случае, дают верные результаты в случае, формально не удовлетворяющем рассматриваемому пределу. Как правило, при расчетах в рамках теории ГЛ используется следующая температурная зависимость $\xi(T)$, применимая вблизи $T_{\mathrm{cm}}$ :

$$
\xi(T)=\frac{\xi(0)}{\sqrt{\left(1-T / T_{\mathrm{cm}}\right)}},
$$

где $\xi(0)-$ длина когерентности при $T=0$.

\section{3. Результаты расчетов и обсуждение}

Вблизи критической температуры цилиндра параметр порядка мал, поэтому кубическим членом в уравнении (3) можно пренебречь, и данное уравнение преобразуется к виду

$$
\frac{\partial^{2} \psi}{\partial \rho^{2}}+\frac{\partial \psi}{\rho \partial \rho}+\psi=0 .
$$

Решением этого уравнения является функция Бесселя нулевого порядка $\left(J_{0}(\rho)\right)$. Граничное условие приводит к соотношению

$$
\frac{\partial J_{0}}{\xi \partial \rho}=-\left.\frac{J_{0}}{\Lambda}\right|_{\rho=\rho_{R}=R / \xi} .
$$

Учитывая свойства функции Бесселя, в пределе $R \ll \xi$ соотношение (7) преобразуется к виду

$$
\left(\frac{R}{\xi}\right)^{2}+\frac{2 \Lambda R}{\xi^{2}}-4=0 .
$$

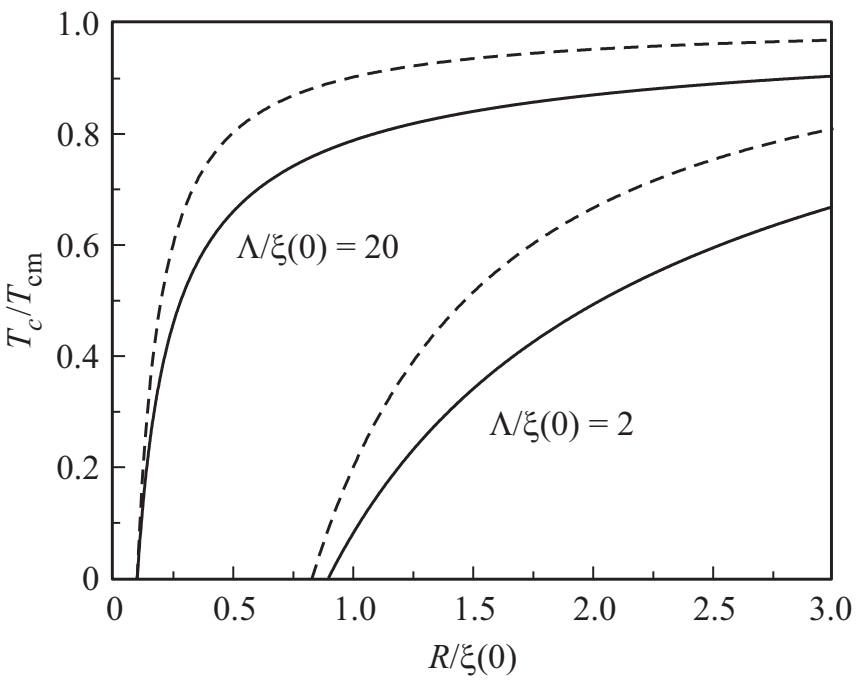

Примеры зависимости критической температуры сверхпроводящего цилиндра от его радиуса для разных значений длины экстраполяции $\Lambda$. Сплошными линиями показаны зависимости, полученные численным решением уравнения (7), а пунктирными показаны зависимости, следующие из соотношения (8), справедливого в пределе $R \ll \xi(0)$.

В дальнейшем мы будем использовать нормированные на $\xi(0)$ единицы длины. Учитывая температурную зависимость длины когерентности (5), получаем соотношение для критической температуры $\left(T_{c}\right)$ цилиндра с радиусом $R$ :

$$
R^{2}+2 \Lambda R=\frac{4}{\left(1-T_{c} / T_{\mathrm{cm}}\right)} .
$$

Преобразуя это соотношение, получаем:

$$
T_{c}=T_{\mathrm{cm}}\left(1-\frac{4}{R(R+2 \Lambda)}\right) .
$$

В общем случае, когда радиус цилиндра сравним с длиной когерентности, зависимость критической температуры цилиндра от его радиуса находится численным решением уравнения (7) и также учитывая температурную зависимость длины когерентности (5). Примеры зависимости критической температуры сверхпроводящего цилиндра от его радиуса для разных значений длины экстраполяции $\Lambda$ показаны на рисунке. Сплошными линиями показаны зависимости, полученные численным решением уравнения (7), а пунктирными зависимости, следующие из соотношения (8), справедливого в пределе $R \ll \xi(0)$. Как видно из рисунка, с уменьшением $R$ и $\Lambda$ влияние граничного условия (4) усиливается, и $T_{c}$ цилиндра уменьшается. Кроме того, с уменьшением радиуса зависимости, следующие из приближенного соотношения (8) становятся близкими к зависимостям, полученным в результате численного решения уравнения (7).

Очевидно, влияние границы на критическую температуру сверхпроводящих цилиндров определяется длиной экстраполяции $\Lambda$. В работах [13-15] был проведен 
микроскопический анализ на основе теории БардинаКупера-Шрифера (БКШ) этого параметра. Для „грязных“ сверхпроводников, в которых длина когерентности БКШ значительно меньше длины свободного пробега электронов, было получено следующее соотношение:

$$
\Lambda=\frac{N(0) V_{e p} \xi^{2}(0)}{a},
$$

где $a$ - радиус экранировки Томаса-Ферми, в металлах его величина близка к постоянной решетки, $N(0)$ - плотность состояний на поверхности Ферми и $V_{e p}$ - энергия электрон-фононного взаимодействия куперовской пары. Произведение $N(0) V_{e p}$ определяет критическую температуру в теории БКШ. Известно, для обычных низкотемпературных сверхпроводников $N(0) V_{e p} \leq 0.3$. Симонин показал [15], что соотношения (2), (9) успешно объясняют уменьшение критической температуры с уменьшением толщины тонких пленок из $\mathrm{Nb}, \mathrm{Pb}$ и $\mathrm{Bi}$. Очевидно, что для уменьшения влияния границ, которое ослабляет сверхпроводимость в тонких пленках и цилиндрах малого диаметра, необходимо увеличивать длину экстраполяции. В согласии с формулой (9), этого можно добиться, создавая соответствующие сверхпроводниковые структуры с меньшим радиусом экранировки $а$ и $c$ более высокими параметрами $N(0), V_{e p}$ и $\xi(0)$.

\section{4. Заключение}

В настоящей работе представлены некоторые результаты численного решения уравнений ГЛ в одномерном случае для цилиндра радиусом порядка длины когерентности $\xi$. Учет влияния границы на сверхпроводящие свойства таких цилиндров, что выражается в применении граничных условий общего вида (4) на параметр порядка с конечными значениями длины экстраполяции $\Lambda$, приводит к уменьшению критической температуры цилиндра. Причем, чем меньше радиус цилиндра и $\Lambda$, тем меньше его критическая температура.

\section{Конфликт интересов}

Автор заявляет, что у него нет конфликта интересов.

\section{Список литературы}

[1] В.Л. Гинзбург, Л.Д. Ландау. ЖЭТФ 10, 1064 (1950).

[2] П.Д. Жен. Сверхпроводимость металлов и сплавов. Мир, M. (1968).

[3] М. Тинкхам. Введение в сверхпроводимость. Атомиздат, M. (1980).

[4] G.J. Carty, D.P. Hampshire. Phys. Rev. B 77, 172501 (2008).

[5] G.R. Berdiyorov, A.K. Elmurodov, F.M. Peeters. Phys. Rev. B 79, 174506 (2009).

[6] G.F. Zharkov, V.G. Zharkov, A.Yu. Zvetkov. Phys. Rev. B 61, 12293 (2000).
[7] А.Ю. Цветков, Г.Ф. Жарков, А.Н. Лыков. Кр. собщения по физике ФИАН, 6, 25 (2004).

[8] А.Н. Лыков, А.Ю. Цветков, Г.Ф. Жарков. ЖЭТФ 128, 392 (2005).

[9] П.И. Безотосный, С.Ю. Гаврилкин, А.Н. Лыков, А.Ю. Цветков. ФТТ 6 (2014).

[10] H.J. Fink, A.G. Presson. Phiys. Rev. 151, 219 (1966).

[11] V.V. Moshchalkov, X.G. Qiu, V. Bruyndoncx. Phys. Rev. B 55, 11793 (1997).

[12] J.M. Calero, N. Porras-Montenegro, J.C. Granada. Braz. J. Phys. 36, 933 (2006).

[13] P.G. De Gennes. Rev. Mod. Phys. 36, 225 (1964).

[14] Р.О. Зайцев. ЖЭТФ 48, 1759 (1965).

[15] J. Simonin. Phys. Rev. B 33, 7830 (1986).

[16] K. Matsumoto, H. Takewaki, Y. Tanaka, O. Miura, K. Yamafuji, K. Funaki, M. Iwakuma, T. Matsushita. Appl. Phys. Lett. 64, 115 (1994).

Редактор Т.Н. Василевская 